\title{
Contrast-enhanced magnetic resonance imaging with a novel nano-size contrast agent for the clinical diagnosis of patients with lung cancer
}

\author{
JIANWEI GAO ${ }^{1,2 *}$, LEI LI ${ }^{3 *}$, XIA LIU ${ }^{2}$, RUI GUO ${ }^{4}$ and BIN ZHAO ${ }^{1}$ \\ ${ }^{1}$ Department of MRI, Shandong Medical Imaging Research Institute, Shandong University, Jinan, \\ Shandong 250021; ${ }^{2}$ Department of Radiology, Tai'an First People's Hospital, Tai'an, Shandong 271000; \\ ${ }^{3}$ Department of Interventional Radiology, The Second Affiliated Hospital of Qingdao University \\ Medical College (Municipal Central Hospital of Qingdao), Qingdao, Shandong 266042; ${ }^{4}$ Department of \\ Gynecology and Obstetrics, Zhangqiu People's Hospital, Zhangqiu, Shandong 250200, P.R. China
}

Received August 12, 2017; Accepted January 8, 2018

DOI: $10.3892 / \mathrm{etm} .2018 .6112$

\begin{abstract}
Recent studies have indicated that magnetic resonance imaging (MRI) efficiently diagnoses lung cancer. However, the efficacy of MRI in diagnosing lung cancer requires improving for patients in the early stage of the disease. In the present study, a novel nano-sized contrast agent of chistosan $/ \mathrm{Fe}_{3} \mathrm{O}_{4}$-enclosed bispecific antibodies (BsAbCENS) was introduced, which targeted carcino-embryonic antigen (CEA) and neuron-specific enolase (NSE) in lung cancer cells. The diagnostic efficacy of contrast-enhanced MRI with BsAbCENS (CEMRI-BsAbCENS) was investigated in a total of 182 patients with suspected lung cancer who had high serum levels of CEA and NSE. BsAbCENS was administered by pulmonary inhalation prior to the MRI scan. The results revealed that CEA and NSE were overexpressed in human lung cancer cell lines. BsAbCENS bound with CEA and NSE on the surface of human lung cancer cells and produced a higher signal intensity than MRI alone for the diagnosis of patients with lung cancer. The diagnostic data revealed that CEMRI-BsAbCENS diagnosed 124/182 lung cancer cases, whereas CEMRI only diagnosed 98/182, which was significantly less $(\mathrm{P}<0.01)$. In addition, the survival rate of patients with lung cancer diagnosed by CEMRI-BsAbCENS was significantly higher than the mean 5 -year survival rate
\end{abstract}

Correspondence to: Professor Bin Zhao, Department of MRI, Shandong Medical Imaging Research Institute, Shandong University, 324 Jingwuweiqi street, Jinan, Shandong 250021, P.R. China

E-mail: zhaobinhospital@aliyun.com

${ }^{*}$ Contributed equally

Key words: lung cancer, diagnosis, magnetic resonance imaging, chistosan $/ \mathrm{Fe}_{3} \mathrm{O}_{4}$-enclosed bispecific antibody, contrast-enhanced magnetic resonance imaging-chistosan $/ \mathrm{Fe}_{3} \mathrm{O}_{4}$-encapsulated bispecific antibodies
$(\mathrm{P}<0.01)$. Furthermore, the pharmacodynamics demonstrated that BsAbCENS was metabolized within $24 \mathrm{~h}$. The results of the present study indicate that the efficacy and accuracy of lung cancer diagnosis are improved by CEMRI-BsAbCENS. In conclusion, these results provide a potential novel protocol for the diagnosis of tumors in patients with suspected early stage lung cancer.

\section{Introduction}

Lung cancer is one of the most prevalent types of human cancer and is the second highest cause of cancer-associated mortality worldwide (1). Previous studies have indicated that lung cancer is typically diagnosed when the disease has reached an advanced stage $(2,3)$. There are different types of lung cancer, including large cell carcinoma, squamous cell carcinoma and adenocarcinoma, and the incidence rates of all types are increasing $(4,5)$. The ability of physicians to diagnose lung cancer using imaging techniques has a substantial impact on the therapeutic decisions that are made and the survival time of patients (6). A missed diagnosis of lung cancer based on imaging tests may deny patients the opportunity to receive effective cancer therapy and result in a diminished survival time (7-9). Therefore, more sensitive methods of cancer diagnosis are required to improve therapeutic options and increase the 5-year survival rate (10).

At present, ultrasound, fludeoxyglucose-positron emission tomography (PET), computed tomography (CT) and magnetic resonance imaging (MRI) are widely used to diagnose and determine the disease stage of human lung cancer $(11,12)$. In a clinical setting, MRI represents the most sensitive and accurate method of diagnosis for lung cancer and metastases (13). A previous study has indicated that the efficacy of contrast-enhanced (CE)MRI in diagnosing brain metastasis in lung cancer is increased compared with regular MRI scanning (14). Additionally, a recent comparison between PET/CT and MRI for diagnosis, staging and follow-up of patients with lung cancer revealed that MRI is useful for distinguishing benign and malignant pulmonary nodules, 
has a higher sensitivity and specificity for nodal staging and is more beneficial in evaluating an early response to systemic chemotherapy (9). Freedman et al (15) have previously demonstrated that nanodelivery of MRI contrast agent (scL-gad-d nanocomplex) enhances the sensitivity of MRI to detect lung cancer metastases. Liposomal-iodinated contrast agent may facilitate the early detection and diagnosis of pulmonary lesions, and have implications on treatment response and monitoring (16). Additionally, although a number of reports have introduced various diagnoses of early-stage lung cancer by CEMRI using a contrast agent, nano-particle sized contrast agents present additional benefits than other contrast agents for the diagnosis of lung cancer, including high sensitivity and specificity (17-19). Therefore, in the present study the auxiliary role of chistosan/ $\mathrm{Fe}_{3} \mathrm{O}_{4}$-encapsulated bispecific antibodies (BsAbCENS) in CEMRI-diagnosed lung cancer was investigated.

At present, tumor markers carcinoembryonic antigen (CEA) and neuron-specific enolase (NSE) are sensitive methods used for the diagnosis and treatment of lung cancer (20). Additionally, the combined detection of hematoporphyrin, CEA, NSE and CYFRA21-1 have been reported to significantly improve the sensitivity and specificity of lung cancer diagnosis, and may be useful for pathological typing (21). In the present study, the efficacy of CEMRI-BsAbCENS in the diagnosis of patients with lung cancer was investigated. It was revealed that CEMRI-BsAbCENS improves the signal intensity at the lung cancer location and also enhances the accuracy and sensitivity of MRI in the diagnosis of clinical patients with suspected lung cancer.

\section{Materials and methods}

Targeted contrast agent. Nano-sized chistosan $/ \mathrm{Fe}_{3} \mathrm{O}_{4}$-enclosed bispecific antibodies (BsAbCENS, obtained from the department of bio-pharmaceuticals, Shandong University, Jinan, China) were produced using the covalent bond method as previously described (22). The bsAbCENS was taken using an atomizer (NE-J01; Contec Medical Systems Co., Ltd., Qinhuangdao, China). It was possible to visualize the nano-particles contrast agent via an MRI system. The BsAbCENS contrast agent was administered via atomizer 30, 60 and $90 \mathrm{~min}$ prior to MRI.

Patients. The inclusion and exclusion criteria of the present study were the same as previously reported (23). A total of 182 patients (104 males and 78 females; mean age, 48.4 years) with suspected early stage lung cancer (NSE $\geq 15 \mu \mathrm{g} / \mathrm{ml}$; CEA $>10 \mu \mathrm{g} / \mathrm{ml}$ ) were enrolled in the present study from Shandong Medical Imaging Research Institute (Jinan, China) between May 2011 and July 2016. Patients were diagnosed according to the European Society for Medical Oncology clinical practice guidelines for the diagnosis of lung cancer (24).

A total of 182 healthy volunteers (105 males, 77 females; mean age, 48.6 years) were recruited from Shandong Medical Imaging Research institute (Jinan, China). All patients and healthy volunteers underwent a CEMRI scan and CEMRI-BsAbCENS for the detection of early-stage lung cancer. The characteristics of all participants within the present study are summarized in Table I.
The experiments in the present study were performed in accordance with the recommendations of the Guide for the Care and Use of Clinical Study of China (approval no. BUCMT20070612M25). The present study was approved by ethics committee of Shandong Medical Imaging Research Institute (Jinan, China) and all patients provided written informed consent prior to their inclusion within the present study.

Cells and reagents. Lung cancer cell line A549 and normal lung cell line MRC-5 were purchased from the American Type Culture Collection (Manassas, VA, USA). A549 cells were cultured in Dulbecco's modified Eagle medium (Sigma-Aldrich; Merck KGaA, Darmstadt, Germany) supplemented with $10 \%$ fetal bovine serum (FBS; Invitrogen; Thermo Fisher Scientific, Inc.). MRC-5 cells were cultured in 1640 medium (Sigma-Aldrich; Merck KGaA) supplemented with $10 \% \mathrm{FBS}$. All cells were cultured at $37^{\circ} \mathrm{C}$ in a $5 \% \mathrm{CO}_{2}$ humidified atmosphere.

ELISA. The serum concentration levels of CEA (cat. no. DY4128), fibroblast growth factor receptor (cat. no. 661FR) and NSE (cat. no. DY5169-05; all R\&D Systems, Inc., Minneapolis, MN, USA) were analyzed by commercialized ELISA kits. The operational procedures were performed according to the manufacturer's protocol. The results were analyzed using an ELISA reader system (Bio-Rad Laboratories, Inc., Hercules, CA, USA).

MRI scanning. An MRI diagnosis system was used to diagnose patients with suspected lung cancer using preprogrammed settings. The settings were optimized to provide the optimal image formation. The whole lungs of all patients in the present study underwent MRI scanning according to the manufacturer's protocol (Ingenia 1.5T CX; Philips Medical Systems, Inc., Bothell, WA, USA). The details of the principles and settings used for MRI scanning were described in a previous study (25).

Image analysis. The outcomes generated by MRI scanning were analyzed using integral software in the MRI machine. Lung cancer location was diagnosed by three physicians using an image produced by the MRI. Patients with lung cancer were analyzed using an MRI scan and the signal enhancement of MRI induced by BsAbCENS was also measured via a pre-prepared program (T1-weighted, T2-weighted and fluid-attenuated inversion recovery sequences) in the MRI machine.

Treatments of patients with lung cancer diagnosed by BsAbCENS-MRI. All Patients with suspected early-stage lung cancer diagnosed via CEMRI-BsAbCENS received different treatments, including chemoradiotherapy, Traditional Chinese Medicine, biological therapy and comprehensive therapy after surgery. The clinical treatment methods for patients with lung cancer are listed in Table II. The median overall survival rate was analyzed as previously described (26).

Immunofluorescence and histological staining. BsAbCENS ( $2 \mathrm{mg} / \mathrm{ml})$ was labeled with fluorescein isothiocyanate (FITC; $1 \mathrm{mg} / \mathrm{ml}$ in dimethylsulphoxide) as described in a 
Table I. Characteristics of study patients.

\begin{tabular}{lcc}
\hline Characteristic & Patients & $\begin{array}{c}\text { Healthy } \\
\text { volunteers }\end{array}$ \\
\hline Male (n) & 104 & 105 \\
Female (n) & 78 & 87 \\
Age range (years) & $28.8-64.6$ & $28.6-64.8$ \\
Medical history of cancer $(\mathrm{n})$ & 8 & 0 \\
Serum CEA $(\mu \mathrm{g} / \mathrm{l})$ & $29.4 \pm 13.3$ & $2.6 \pm 1.8$ \\
Serum NSE $(\mu \mathrm{g} / \mathrm{ml})$ & $27.8 \pm 15.0$ & $7.8 \pm 4.1$ \\
\hline
\end{tabular}

CEA, carcino-embryonic antigen.

Table II. Treatment of patients with lung cancer.

\begin{tabular}{lcc}
\hline Treatment & Male $(\mathrm{n}=78)$ & Female $(\mathrm{n}=52)$ \\
\hline Chemoradiotherapy & 40 & 27 \\
Chinese medicine & 10 & 13 \\
Biological therapy & 14 & 5 \\
Comprehensive therapy & 14 & 7 \\
following surgery & & \\
\hline
\end{tabular}

previous study (27). Prior to immunofluorescence staining, A549 and MRC-5 cells were cultured to $85 \%$ confluence. All cells were fixed with $10 \%$ paraformaldehyde for $30 \mathrm{~min}$ at $37^{\circ} \mathrm{C}$ and subsequently incubated with rabbit anti-human FITC-labeled antibodies targeting CEA (1:1,000; cat. no. ab133633, Abcam, Cambridge, UK) or NSE (1:1,000; cat. no. ab53025; Abcam) for $1 \mathrm{~h}$ at $25^{\circ} \mathrm{C}$. The cells were washed three times with PBS and observed using a fluorescence microscope (CKX53; Olympus Corporation, Tokyo, Japan). For histological staining $4-\mu \mathrm{m}$-thick tumor sections were fixed with $10 \%$ formaldehyde for $10 \mathrm{~min}$ at $37^{\circ} \mathrm{C}$ and then stained with hematoxylin and eosin for $1 \mathrm{~h}$ at $37^{\circ} \mathrm{C}$ as previously described (28).

Statistical analysis. All data are presented as the mean \pm standard deviation of 3 experiments. Unpaired data was analyzed using Student's t-test. Kaplan-Meier analysis was used to estimate the survival rate every 5-month call visits during 60 months observation (to observe overall survival rate and tumor recurrence). $\mathrm{P}<0.05$ was considered to indicate a statistically significant difference.

\section{Results}

Characteristics of patients. A total of 182 patients with suspected lung cancer and 182 age-matched healthy volunteers were enrolled in the present study. The age range was 28.8-64.6 and 28.6-64.8 years in the patients and healthy volunteers, respectively. The numbers of male and female participants were similar in the patient and healthy volunteer groups. The characteristics of all patients included in the present study are summarized in Table I.
Analysis of the serum expression of CEA and NSE in lung cancer tissues and normal cells. The serum levels of CEA and NSE were analyzed in patients with lung cancer and healthy volunteers. It was observed that the serum levels of CEA and NSE were significantly higher in patients with lung cancer compared with healthy individuals (Fig. 1A and B). It was demonstrated that CEA and NSE were overexpressed in lung cancer tissues compared with the adjacent normal tissues (Fig. 1C). Immunofluorescence analysis revealed that the expression levels of CEA and NSE were upregulated in the A549 lung cancer cell line compared with the MRC-5 normal lung cell line (Fig. 1D). It was also demonstrated that BsAbCENS notably decreased the CEA and NSE expression levels in lung cancer cells (Fig. 1E). These results indicate that patients with lung cancer exhibited higher plasma CEA and NSE levels than healthy volunteers.

Efficacy of CEMRI-BsAbCENS in early clinical diagnosis for patients with suspected lung cancer. The diagnostic efficacy of CEMRI-BsAbCENS was investigated in patients with suspected lung cancer. The dose of CEMRI-BsAbCENS that provided the optimal signal intensity for CEMIR detection was $24 \mathrm{mg} / \mathrm{kg}$ for $30 \mathrm{~min}$ (Fig. 2A). CEMRI-BsAbCENS diagnosed 122 (68.13\%; 76 male and 46 female) patients with lung cancer, whereas CEMRI diagnosed 98 (53.85\%; 62 male and 36 female) patients with lung cancer. This demonstrated that CEMRI-BsAbCENS diagnosed a significantly higher number of patients with lung cancer compared with CEMRI (Fig 2B). Following $60 \mathrm{~min}$ inhalation of BsAbCENS it was also observed that there were significantly decreased serum concentrations of CEA and NSE in patients with lung cancer compared with those who had not inhaled BsAbCENS (Fig. 2C and D). These results suggest that CEMRI-BsAbCENS is more effective than CEMRI due to binding with CEA and NSE for the diagnosis of lung cancer than CEMRI.

Histopathology confirms the diagnosis of CEMRI-BsAbCENS for lung cancer cases. Histopathology images were used to further confirm the results of the CEMRI-BsAbCENS and CEMRIs. It was demonstrated that the BsAbCENS contrast agent improved image quality generated by MRI (Fig. 3A). The representative images demonstrate that CEMRI-BsAbCENS defined the image of the tumor more clearly than the CEMRI (Fig. 3B). The CEMRI-BsAbCENS diagnosis of patients with lung cancer was confirmed by histological analysis of lung tissues (Fig. 3C). Histopathology confirmed that 130 (78 male patients and 52 female patients) patients had lung cancer and CEMRI-BsAbCENS diagnosed 124 of these patients. The diagnostic rate was significantly higher for CEMRI-BsAbCENS compared with CEMRI for patients with suspected lung cancer (Fig. 3D). These results suggest that CEMRI-BsAbCENS is accurate in diagnosing patients with lung cancer.

Pharmacodynamics of BsAbCENS in the serum of patients with lung cancer. The signal intensity of CEMRI-BsAbCENS following the administration of BsAbCENS was investigated in patients with lung cancer. The results revealed that BsAbCENS was almost fully metabolized from the blood at $24 \mathrm{~h}$ following 
A

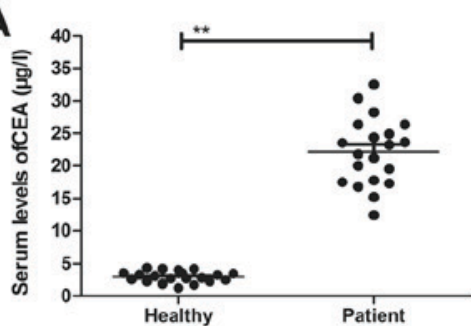

B

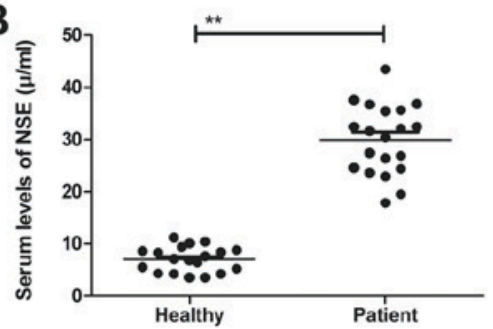

C Adjacent tissues

CEA

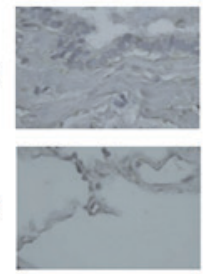

Tumor tissue

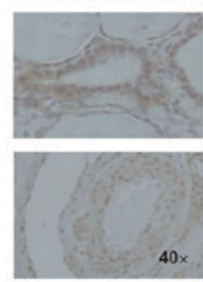

D

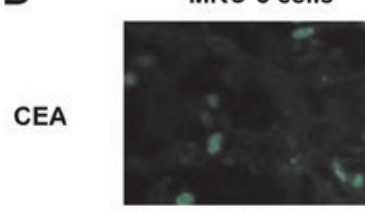

NSE

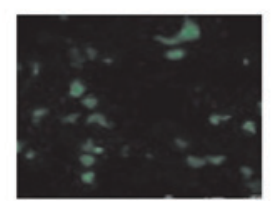

A549 cells
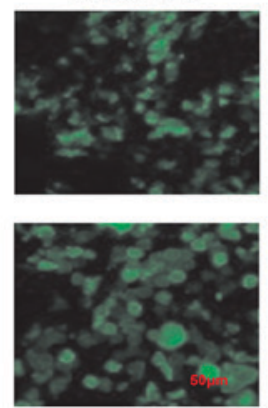

$\mathbf{E}$

CEA

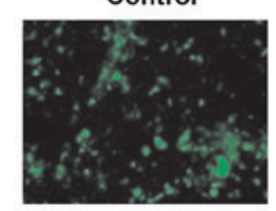

NSE

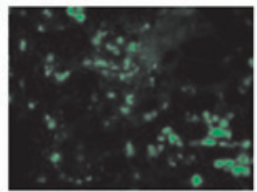

BsAbCENS
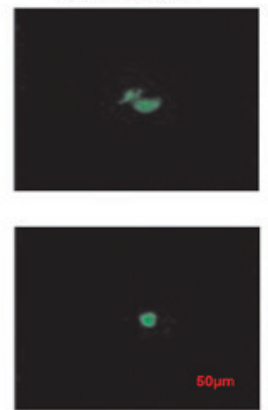

Figure 1. Analysis of the plasma expression of CEA and NSE in lung cancer tissues and normal cells. The serum levels of (A) CEA and (B) NSE were measured in patients with lung cancer and healthy individuals. (C) Representative CEA and NSE expression pictures of lung cancer tissue compared with adjacent normal tissues. Magnification, x40. (D) Immunofluorescence analysis of the expression levels of CEA and NSE in a lung cancer cell line (A549) and normal lung cell line (MRC-5). (E) The neutralization role of BsAbCENS on CEA and NSE expression levels in lung cancer cells. " $\mathrm{P}<0.01$ vs. CEMRI. CEA, carcino-embryonic antigen; NSE, neuron-specific enolase; BsAbCENS, chistosan $/ \mathrm{Fe}_{3} \mathrm{O}_{4}$-enclosed bispecific antibodies.

A

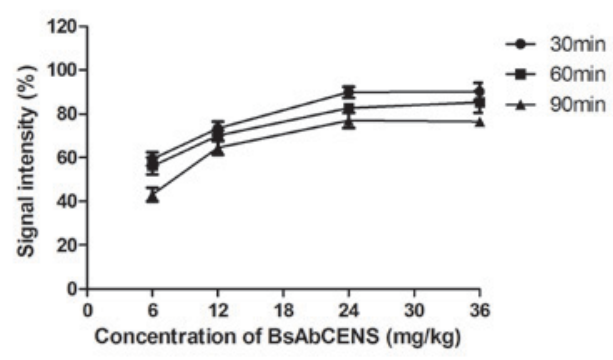

C

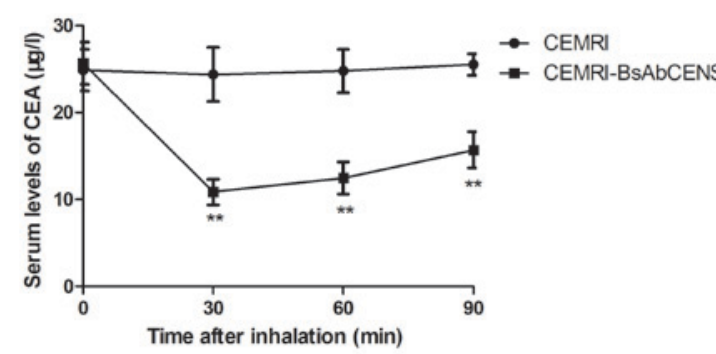

B

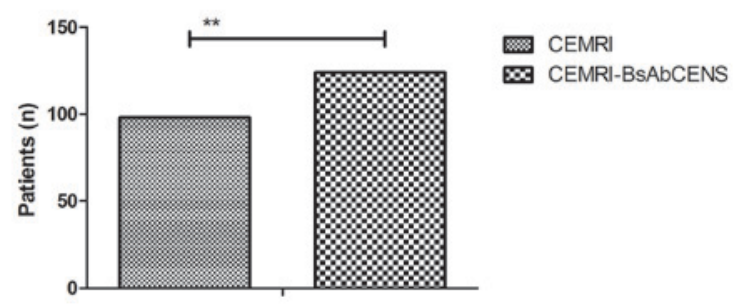

D

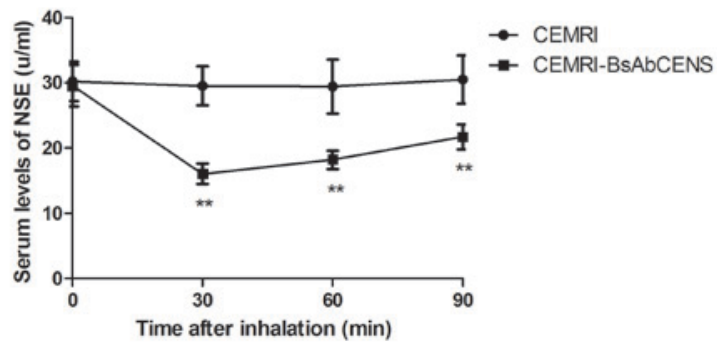

Figure 2. Efficacy of CEMRI-BsAbCENS for the diagnosis of patients with suspected lung cancer. (A) Dose determination of the signal intensity of CEMRI-BsAbCENS for clinical patients. (B) The efficacy of CEMRI-BsAbCENS and CEMRI for the diagnosis of patients with lung cancer was determined. Serum levels of (C) CEA and (D) NSE in patients with lung cancer following the inhalation of BsAbCENS. ${ }^{* *} \mathrm{P}<0.01$ vs. CEMRI. CEA, carcino-embryonic antigen; NSE, neuron-specific enolase; CEMRI, contrast-enhanced magnetic resonance imaging; BsAbCENS, chistosan/Fe $\mathrm{O}_{3}$-enclosed bispecific antibodies.

inhalation (Fig. 4A). It was observed that patients diagnosed with lung cancer by CEMRI-BsAbCENS had a significantly improved survival rate compared with the mean 5-year survival rate (Fig. 4B). These results indicate that BsAbCENS increased the signal intensity for CEMRIs in patients with lung cancer.

\section{Discussion}

MRI is a type of imaging technique that is currently available for the noninvasive diagnosis of human carcinomas $(29,30)$. CEMRI has been demonstrated to be more effective than 
A

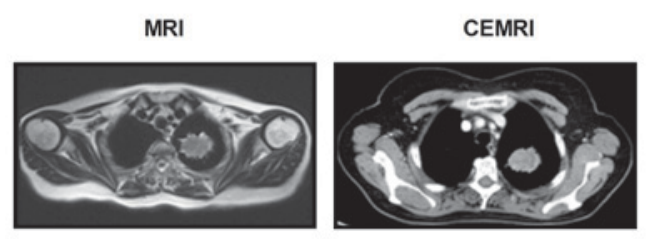

C

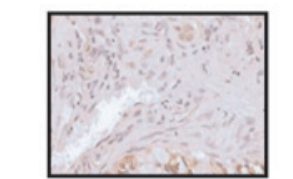

Squamous cell carcinoma

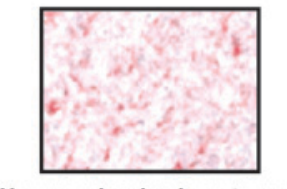

Neuroendocrine lung tumor

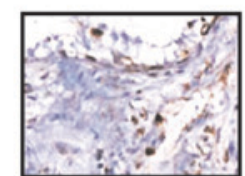

Adenocarcinoma

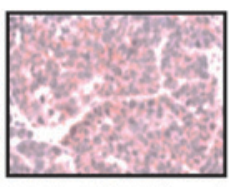

B
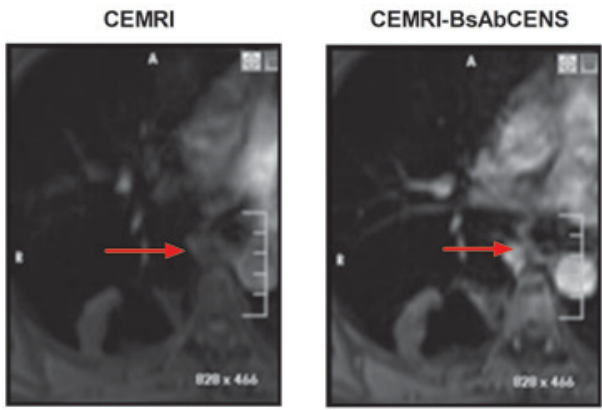

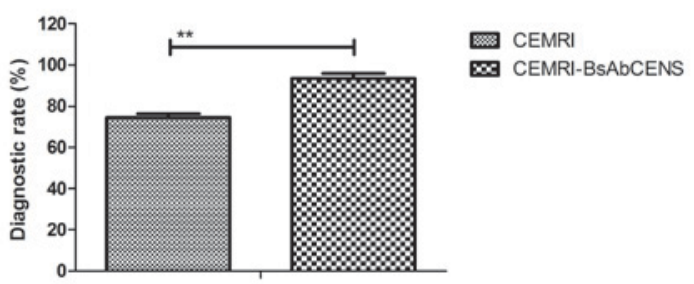

Figure 3. Histopathology analyses of the diagnostic outcomes of CEMRI-BsAbCENS for patients with lung cancer. (A) The BsAbCENS contrast agent increased the image quality generated by MRI. (B) Representative MRI images generated by CEMRI and CEMRI-BsAbCENS for patients with lung cancer. Red arrows indicate tumor location. (C) Representative histological analyses of lung tissues in patients with lung cancer. (D) The diagnostic rate of CEMRI-BsAbCENS and CEMRI for patients with suspected lung cancer. MRI, magnetic resonance imaging; CEMRI, contrast-enhanced magnetic resonance imaging; BsAbCENS, chistosan/ $\mathrm{Fe}_{3} \mathrm{O}_{4}$-enclosed bispecific antibodies.
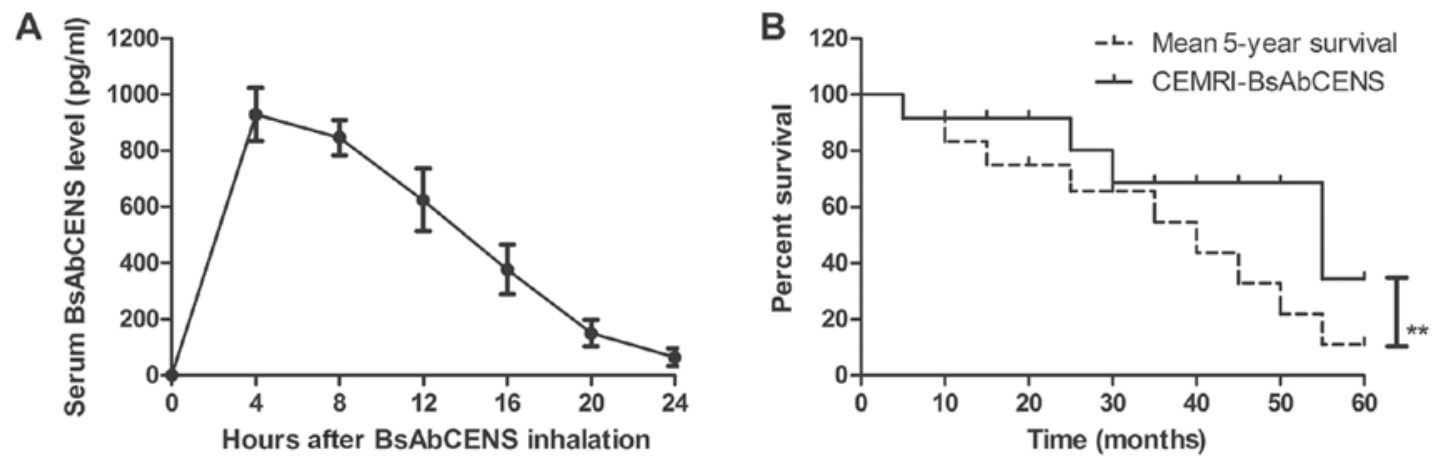

Figure 4. Pharmacodynamics of BsAbCENS in the serum of patients with lung cancer. (A) The metabolic cycle of BsAbCENS in the serum of patients with lung cancer. (B) The survival rate of patients diagnosed with lung cancer by CEMRI-BsAbCENS compared with the mean 5-year survival rate. CEMRI, contrast-enhanced magnetic resonance imaging; BsAbCENS, chistosan $/ \mathrm{Fe}_{3} \mathrm{O}_{4}$-enclosed bispecific antibodies .

MRI in differentiating between melanoma and lung cancer brain metastases (31). In the present study, the diagnostic efficacy of CEMRI-BsAbCENS was further analyzed in a total of 182 patients with suspected lung cancer who had high serum levels of CEA and NSE. The results revealed that contrast agent BsAbCENS was able to bind with CEA and NSE on lung tumor cells and present a higher signal intensity than MRI in the same patients. CEMRI-BsAbCENS markedly improved sensitivity and significantly improved the diagnostic accuracy for patients with suspected lung cancer compared with MRI.
Early diagnosis of lung cancer allows for the implementation of cancer treatments that may improve the overall survival rate of patients (32-34). A previous study has demonstrated that CEMRI was able to identify patients who would benefit from bevacizumab and erlotinib treatment compared with CT based on molecular imaging for an earlier diagnosis (35). The present study reported that CEMRI-BsAbCENS diagnosed $124 / 182$ patients with lung cancer and significantly improved the sensitivity and specificity of diagnosis. Nensa et al (36) have previously suggested that dynamic CEMRI parameters may act as biomarkers for the therapeutic effects of 
vatalanib in patients with non-small-cell lung cancer. The present study has revealed that BsAbCENS binds with the lung cancer biomarkers CEA and NSE, which increased the signal intensity produced by MRI. Lung cancer patients had a higher survival rate following diagnosis by CEMRI-BsAbCENS compared with the mean 5-year survival rate, suggesting that CEMRI-BsAbCENS has a potential application for the early diagnosis of lung cancer. However, patients received different anti-cancer treatments, which may have affected survival rate, thus making it a limitation of this study.

Serum CEA is higher in patients with small cell lung cancer and non-small-cell lung cancer and has been used to improve the diagnostic sensitivity for human lung cancer $(20,37)$. The clinical use of NSE has significantly improved the sensitivity and specificity of lung cancer diagnosis and may be useful for pathological typing (21). The combination of CEA and NSE may be an effective clinical confirmation and exclusive diagnostic indictor of meningeal carcinomatosis in lung cancer (38). In the present study, it was observed that BsAbCENS targeted CEA and NSE on lung cancer cells. A previous study has indicated that MRI contrast agent was able to detect an early stage glioma (39). In addition, fibronectin-targeting contrast agent in combination with MRI may detect breast cancer micrometastases (40). The present study revealed that BsAbCENS, when used with MRI, significantly improved the diagnostic accuracy and sensitivity for patients with lung cancer. Furthermore, contrast-agent MRI targeting of CA1 may produce high-resolution MR molecular imaging of human lung adenocarcinoma A549 cells (41). The results of the present study revealed that CEMRI-BsAbCENS was more effective than MRI for the diagnosis of patients with lung cancer. However, stratification for NSE $\geq 15 \mu / \mathrm{ml}$ was not conducted for patients with suspected lung cancer, which indicates a potential bias in the analysis.

In conclusion, the present study has described a novel approach for improved diagnostic accuracy of patients with lung cancer within a clinical setting, using MRI images with a targeting contrast agent. The effect of this approach on the overall survival of patients with lung cancer has also been discussed. These results suggest that CEMRI-BsAbCENS may present a significant clinical advantage to clinicians diagnosing patients with suspected lung cancer. However, further research should be conducted with a larger cohort to confirm these findings.

\section{Acknowledgements}

Not applicable.

\section{Funding}

No funding was received.

\section{Availability of data and materials}

The analyzed data sets generated during the present study are available from the corresponding author on reasonable request.

\section{Authors' contributions}

JG, LL, Xl and RG performed the experiments and BZ designed and analyzed the data of the present study.

\section{Ethics approval and consent to participate}

The experiments in the present study were performed in accordance with the recommendations of the Guide for the Care and Use of Clinical Study of China (approval no. BUCMT20070612M25). The present study was approved by ethics committee of Shandong Medical Imaging Research Institute (Jinan, China) and all patients provided written informed consent prior to their inclusion within the present study.

\section{Consent for publication}

Not applicable.

\section{Competing interests}

The authors declare that they have no competing interests.

\section{References}

1. Zhou C and Yao LD: Strategies to improve outcomes of patients with EGRF-mutant non-small cell lung cancer: Review of the literature. J Thorac Oncol 11: 174-186, 2016.

2. Zhang H, Gao L, Zhang B, Zhang L and Wang C: Prognostic value of platelet to lymphocyte ratio in non-small cell lung cancer: A systematic review and meta-analysis. Sci Rep 6: 22618, 2016.

3. Zhao S, Jiang T, Zhang L, Yang H, Liu X, Jia Y and Zhou C: Clinicopathological and prognostic significance of regulatory $\mathrm{T}$ cells in patients with non-small cell lung cancer: A systematic review with meta-analysis. Oncotarget 7: 36065-36073, 2016.

4. Tomos I, Vlami A, Karakatsani A, Korbila I, Manali ED and Papiris SA: Diffuse idiopathic skeletal hyperostosis (DISH) and non small cell lung cancer: Case presentation and review of the literature. Pneumonol Alergol Pol 84: 116-118, 2016.

5. van der Wekken AJ, Saber A, Hiltermann TJ, Kok K, van den Berg A and Groen HJ: Resistance mechanisms after tyrosine kinase inhibitors afatinib and crizotinib in non-small cell lung cancer, a review of the literature. Crit Rev Oncol Hematol 100: 107-116, 2016.

6. Folch E, Costa DB, Wright J and VanderLaan PA: Lung cancer diagnosis and staging in the minimally invasive age with increasing demands for tissue analysis. Transl Lung Cancer Res 4: 392-403, 2015.

7. Ju F, Lee HK, Osarogiagbon RU, Yu X, Faris N and Li J: Computer modeling of lung cancer diagnosis-to-treatment process. Transl Lung Cancer Res 4: 404-414, 2015.

8. Khan M, Wasim A, Mirrakhimov AE, McMahon BA, Judge DP Chu LC, Banavali A and Zeidan AM: Case report of a patient with left ventricular assistance device undergoing chemotherapy for a new diagnosis of lung cancer. Case Rep Oncol Med 2015: $163727,2015$.

9. Kim HS, Lee KS, Ohno Y, van Beek EJ and Biederer J: PET/CT versus MRI for diagnosis, staging, and follow-up of lung cancer. J Magn Reson Imaging 42: 247-260, 2015.

10. Blumenthal GM, Karuri SW, Zhang H, Zhang L, Khozin S, Kazandjian D, Tang S, Sridhara R, Keegan P and Pazdur R: Overall response rate, progression-free survival, and overall survival with targeted and standard therapies in advanced non-small-cell lung cancer: US food and drug administration trial-level and patient-level analyses. J Clin Oncol 33: 1008-1014, 2015.

11. Jimenez-Bonilla JF, Quirce R, Martinez-Rodriguez I, De Arcocha-Torres M, Carril JM and Banzo I: The role of PET/CT molecular imaging in the diagnosis of recurrence and surveillance of patients treated for non-small cell lung cancer. Diagnostics (Basel) 6: E36, 2016. 
12. Baco E, Rud E, Ukimura O, Vlatkovic L, Svindland A Matsugasumi T, Bernhard JC, Rewcastle JC and Eggesbø HB: Effect of targeted biopsy guided by elastic image fusion of MRI with 3D-TRUS on diagnosis of anterior prostate cancer. Urol Oncol 32: 1300-1307, 2014.

13. Krüger S, Mottaghy FM, Buck AK, Maschke S, Kley H, Frechen D, Wibmer T, Reske SN and Pauls S: Brain metastasis in lung cancer. Comparison of cerebral MRI and 18F-FDG-PET/CT for diagnosis in the initial staging. Nuklearmedizin 50: 101-106, 2011.

14. Li ZL, Chen X, Xia CC, Sun JY, Li CX, Tang HH and Song B: Comparison of the efficacy of gadobutrol and multihance in contrast-enhanced MRI for diagnosis of brain metastasis in lung cancer. Sichuan Da Xue Xue Bao Yi Xue Ban 43: 601-604, 2012 (In Chinese).

15. Freedman M, Chang EH, Zhou Q and Pirollo KF: Nanodelivery of MRI contrast agent enhances sensitivity of detection of lung cancer metastases. Acad Radiol 16: 627-637, 2009.

16. Badea CT, Athreya KK, Espinosa G, Clark D, Ghafoori AP, Li Y, Kirsch DG, Johnson GA, Annapragada A and Ghaghada KB: Computed tomography imaging of primary lung cancer in mice using a liposomal-iodinated contrast agent. PloS One 7: e34496, 2012 .

17. Pauls S, Mottaghy FM, Schmidt SA, Krüger S, Möller P, Brambs HJ and Wunderlich A: Evaluation of lung tumor perfusion by dynamic contrast-enhanced MRI. Magn Reson Imaging 26: 1334-1341, 2008.

18. Mistry NN, Pollaro J, Song J, De Lin M and Johnson GA: Pulmonary perfusion imaging in the rodent lung using dynamic contrast-enhanced MRI. Magn Reson Med 59: 289-297, 2008.

19. Eichinger M, Puderbach M, Fink C, Gahr J, Ley S, Plathow C, Tuengerthal S, Zuna I, Müller FM and Kauczor HU: Contrast-enhanced 3D MRI of lung perfusion in children with cystic fibrosis--initial results. Eur Radiol 16: 2147-2152, 2006.

20. Lazarev SM, Massard Zh, Reshetov AV, Nikolaev GV, Volgin GN, Osipov EV, Lomteva EIu, Nokhrin AV and Kakysheva OE: Role of biological tumor markers CEA, Cyfra-21, NSE, TU M2-PK in diagnosis and treatment of lung cancer. Vestn Khir Im I I Grek 169: 39-43, 2010 (In Russian).

21. Wang B, He YJ, Tian YX, Yang RN, Zhu YR and Qiu H: Clinical utility of haptoglobin in combination with CEA, NSE and CYFRA21-1 for diagnosis of lung cancer. Asian Pac J Cancer Prev 15: 9611-9614, 2014.

22. Chen CL, Hu GY, Mei Q, Qiu H, Long GX and Hu GQ: Epidermal growth factor receptor-targeted ultra-small superparamagnetic iron oxide particles for magnetic resonance molecular imaging of lung cancer cells in vitro. Chin Med J (Engl) 125: 2322-2328, 2012

23. Preskorn SH, Macaluso M and Trivedi M: How commonly used inclusion and exclusion criteria in antidepressant registration trials affect study enrollment. J Psychiatr Pract 21: 267-274, 2015

24. Crinò L, Weder W, van Meerbeeck J, Felip E and ESMO Guidelines Working Group: Early stage and locally advanced (non-metastatic) non-small-cell lung cancer: ESMO clinical practice guidelines for diagnosis, treatment and follow-up. Ann Oncol 21 Suppl 5: v103-v115, 2010.

25. Sahibzada I, Batura D and Hellawell G: Validating multiparametric MRI for diagnosis and monitoring of prostate cancer in patients for active surveillance. Int Urol Nephrol 48: 529-533, 2016.

26. Boehm K, Schiffmann J, Tian Z, Lesmana H, Larcher A Mandel P, Karakiewicz PI, Graefen M, Schwarz R, Krüll A and Tilki D: Five-year biochemical recurrence-free and overall survival following high-dose-rate brachytherapy with additional external beam or radical prostatectomy in patients with clinically localized prostate cancer. Urol Oncol 34: 119.e11-e18, 2016.

27. Zhao JJ, Chen J, Wang ZP, Pan J and Huang YH: Double labeling and comparison of fluorescence intensity and photostability between quantum dots and FITC in oral tumors. Mol Med Rep 4: 425-429, 2011
28. Kargahi N, Razavi SM, Deyhimi P and Homayouni S: Comparative evaluation of eosinophils in normal mucosa, dysplastic mucosa and oral squamous cell carcinoma with hematoxylin-eosin, Congo red, and EMR1 immunohistochemical staining techniques. Electron Physician 7: 1019-1026, 2015.

29. Ocak I, Bernardo M, Metzger G, Barrett T, Pinto P, Albert PS and Choyke PL: Dynamic contrast-enhanced MRI of prostate cancer at 3 T: A study of pharmacokinetic parameters. AJR Am J Roentgenol 189: 849, 2007.

30. Rappeport ED, Loft A, Berthelsen AK, von der Recke P, Larsen PN, Mogensen AM, Wettergren A, Rasmussen A, Hillingsoe J, Kirkegaard P and Thomsen C: Contrast-enhanced FDG-PET/CT vs. SPIO-enhanced MRI vs. FDG-PET vs. CT in patients with liver metastases from colorectal cancer: A prospective study with intraoperative confirmation. Acta Radiol 48: 369-378, 2007.

31. Hatzoglou V, Tisnado J, Mehta A, Peck KK, Daras M, Omuro AM, Beal K and Holodny AI: Dynamic contrast-enhanced MRI perfusion for differentiating between melanoma and lung cancer brain metastases. Cancer Med 6: 761-767, 2017.

32. Ye ZJ, Qiu HZ, Li PF, Liang MZ, Zhu YF, Zeng Z, Hu GY, Wang SN and Quan XM: Predicting changes in quality of life and emotional distress in chinese patients with lung, gastric, and colon-rectal cancer diagnoses: The role of psychological resilience. Psychooncology 26: 829-835, 2017.

33. Kaseda K, Watanabe K, Asakura K, Kazama A and Ozawa Y: Identification of false-negative and false-positive diagnoses of lymph node metastases in non-small cell lung cancer patients staged by integrated (18F-)fluorodeoxyglucose-positron emission tomography/computed tomography: A retrospective cohort study. Thorac Cancer 7: 473-480, 2016.

34. Abraha I, Giovannini G, Serraino D, Fusco M and Montedori A: Validity of breast, lung and colorectal cancer diagnoses in administrative databases: A systematic review protocol. BMJ Open 6: e010409, 2016.

35. De Langen AJ, van den Boogaart V, Lubberink M, Backes WH, Marcus JT, van Tinteren H, Pruim J, Brans B, Leffers P, Dingemans AM, et al: Monitoring response to antiangiogenic therapy in non-small cell lung cancer using imaging markers derived from PET and dynamic contrast-enhanced MRI. J Nucl Med 52: 48-55, 2011

36. Nensa F, Stattaus J, Morgan B, Horsfield MA, Soria JC, Besse B, Gounant V, Khalil A, Seng K, Fischer B, et al: Dynamic contrast-enhanced MRI parameters as biomarkers for the effect of vatalanib in patients with non-small-cell lung cancer. Future Oncol 10: 823-833, 2014.

37. Zhao W, Yu H, Han Z, Gao N, Xue J and Wang Y: Clinical significance of joint detection of serum CEA, SCCA, and bFGF in the diagnosis of lung cancer. Int J Clin Exp Pathol 8: 9506-9511, 2015.

38. Wang P, Piao Y, Zhang X, Li W and Hao X: The concentration of CYFRA 21-1, NSE and CEA in cerebro-spinal fluid can be useful indicators for diagnosis of meningeal carcinomatosis of lung cancer. Cancer Biomark 13: 123-130, 2013.

39. Liu X, Madhankumar AB, Miller PA, Duck KA, Hafenstein S, Rizk E, Slagle-Webb B, Sheehan JM, Connor JR and Yang QX: MRI contrast agent for targeting glioma: Interleukin-13 labeled liposome encapsulating gadolinium-DTPA. Neuro Oncol 18: 691-699, 2016.

40. Zhou Z, Qutaish M, Han Z, Schur RM, Liu Y, Wilson DL and Lu ZR: MRI detection of breast cancer micrometastases with a fibronectin-targeting contrast agent. Nat Commun 6: 7984, 2015.

41. Yang Y, Zhou J and Yu K: Design, synthesis, and in vitro evaluation of a binary targeting MRI contrast agent for imaging tumor cells. Amino Acids 46: 449-457, 2014. 